\title{
A DISCRETE KPP-THEORY FOR FISHER'S EQUATION
}

\author{
BENGT HAKBERG
}

\begin{abstract}
The purpose of this paper is to extend the theory by Kolmogorov, Petrowsky and Piscunov (KPP) for Fisher's equation, to a discrete solution. We approximate the time derivative in Fisher's equation by an explicit Euler scheme and the diffusion operator by a symmetric difference scheme of second order. We prove that the discrete solution converges towards a traveling wave, under restrictions in the time- and space-widths, as the number of time steps increases to infinity. We also prove that the flame velocity can be determined as a solution to an optimization problem.
\end{abstract}

\section{INTRODUCTION}

Consider the initial-value problem

$$
\begin{aligned}
\partial u / \partial t & =\partial^{2} u / \partial x^{2}+F(u),-\infty<x<\infty, t>0, \\
u(x, 0) & =f(x),-\infty<x<\infty,
\end{aligned}
$$

where $F(u)$ is a smooth function, which satisfies the conditions

$$
\begin{aligned}
& F(0)=F(1)=0, \\
& F(u)>0, \text { for } 0<u<1, \\
& F^{\prime}(0)=1 \text { and } F(u) \leq F^{\prime}(0) u=u, \text { for } 0<u \leq 1 .
\end{aligned}
$$

The equation (1.1) was proposed by Fisher [1] as a model for propagation of an advantageous gene population in an infinite region.

A mathematical theory for Fisher's equation, with the initial condition

$$
f(x)=0, x<0 \text { and } f(x)=1, x>0,
$$

was formulated by Kolmogorov, Petrowsky and Piscunow [4]. This theory (the KPP-theory) has been used extensively in combustion studies; see Hakberg 2 .

The problem of numerical validation of an extended KPP-theory for models in turbulent combustion, motivates the study of the discrete flame velocity for Fisher's equation.

Suppose that we approximate Fisher's equation (1.1) with the explicit difference scheme

$$
\begin{aligned}
& (v(x,(n+1) k)-v(x, n k)) / k=(v(x-h, n k)-2 v(x, n k) \\
& \quad+v(x+h, n k)) / h^{2}+F(v(x, n k)),-\infty<x<\infty, \forall n \geq 0,
\end{aligned}
$$

where $h$ and $k$ are mesh-widths in $x$ and $t$, respectively, and $v(x, n k)$ is an approximation of $u(x, n k)$. The initial-condition (1.6) is approximated by

$$
v(x, 0)=0, x<0 ; v(x, 0)=x / h, 0 \leq x \leq h \text { and } v(x, 0)=1, h<x .
$$

Received by the editor December 6, 2010 and, in revised form, October 11, 2011. 2010 Mathematics Subject Classification. Primary 65M06.

(C) 2012 American Mathematical Society Reverts to public domain 28 years from publication 
Let $c$ be a fixed, but arbitrary constant, such that $0<c<1$, and let $\varphi^{n}$ be defined by

$$
v\left(\varphi^{n}, n k\right)=c, \forall n \geq 0,
$$

which is possible, since $v(x, n k)$ is continuous for all $x$ and strictly monotone for $0<v(x, n k)<1$, if $2 \lambda+k \kappa<1$, where $\kappa=\sup _{0<u<1}\left|F^{\prime}(u)\right|$ and

$$
\lambda=k / h^{2} .
$$

See Theorem 1.

The first step in the proof of convergence of the discrete solution $v(x, n k)$ towards a traveling flame, is to show that $v\left(\varphi^{n}+i h, n k\right)$ is a monotone function of $n$ for fixed $i$. This result is valid according to Theorem 3 , if $3 \lambda+k \kappa<1$.

Assume that the conditions in Theorem 3 are satisfied. Define the function $v^{*}(x, n k)$ by

$$
v^{*}(x, n k)=v\left(x+\varphi^{n}, n k\right),
$$

where $\varphi^{n}$ is defined by (1.9). Then

$$
v^{*}(0, n k)=v\left(\varphi^{n}, n k\right)=c,
$$

and

exists. See Theorem 4.

$$
\lim _{n \rightarrow \infty} v^{*}(i h, n k)=v^{*}(i h), \forall i \in Z
$$

The function

$$
v_{m}(i h, n k)=v\left(i h+\varphi^{m},(n+m) k\right), \forall i, \forall n \geq 0, \forall m \geq 0,
$$

converges as $m \rightarrow \infty$, towards a function $V(i h, n k)$, which satisfies the difference equation

$$
\begin{aligned}
& V(i h,(n+1) k)=(1-2 \lambda) V(i h, n k)+\lambda V(i h-h, n k))+\lambda V(i h+h, n k) \\
& \quad+k F(V(i h, n k)), \forall i, \forall n \geq 0,
\end{aligned}
$$

with initial data

$$
V(i h, 0)=v^{*}(i h), \forall i
$$

where $v^{*}(i h)$ is defined above. See Theorem 5 .

Numerical experiments suggest that the discrete flame velocity, defined as $\left(\varphi^{n+1}-\varphi^{n}\right) / k$, fluctuates and does not converge as $n \rightarrow \infty$. Theorem 6 shows that the mean flame velocity, defined as $\varphi^{n} / n k$, converges as $n \rightarrow \infty$ to a limit

$$
\lim _{n \rightarrow \infty} \varphi^{n} / n k=\gamma h / k, \text { with } h \text { and } k \text { fixed. }
$$

The result is derived under the restrictions $3 \lambda+k \kappa<1$, where

$$
\kappa=\sup _{0<u<1}\left|F^{\prime}(u)\right| \text {. }
$$

Let us first consider the case, when $\gamma$ is a rational number. Let

$$
\gamma=P / Q
$$

where $P$ and $Q>0$ are integers with no common factor. From the proof of Theorem 7 it follows that

$$
V((i+P) h,(j+Q) k)=V(i h, j k), \forall i, \forall j \geq 0 .
$$

If we introduce a function $\mathrm{W}$, defined by

$$
W(i h-j h P / Q)=V(i h, j k), \forall i, \forall j \geq 0,
$$


then $W$ satisfies the traveling wave equation

$$
\begin{aligned}
& W(i h-(j+1) h P / Q)=(1-2 \lambda) W(i h-j h P / Q)+\lambda W(i h-h-j h P / Q) \\
& +\lambda W(i h+h-j h P / Q)+k F(W(i h-j h P / Q)), \forall i, \forall j \geq 0 .
\end{aligned}
$$

In Theorem 8 , we prove a similar result, if $\gamma$ is an irrational number.

In Theorem 9 , we prove that $\gamma$ can be determined from the optimization problem

$$
\gamma=-\inf _{\alpha>0}\{\log [1-2 \lambda+k+\lambda(\exp (\alpha h)+\exp (-\alpha h))] /(\alpha h)\},
$$

based upon an upper estimate of the discrete flame velocity, proved in Theorem 2.

The analysis shows that the discrete equation (1.7) has a traveling wave solution $v(x-n \gamma h)$, and no traveling wave solution exists, which has a smaller flame velocity in absolute value.

We therefore conclude that the discrete flame converges towards a traveling flame. The convergence is both in shape or form, defined by Theorem 4 and in velocity, defined by Theorem 6 .

A general theory for discrete approximations of Fisher's equation has been formulated by Weinberger [5. The asymptotic flame velocity agrees with $\gamma h / k$ for the explicit difference scheme (1.7), if $2 \lambda+k \kappa<1$. The initial function in Weinberger [5] is a pulse compared to a step in Kolmogorov, Petrowsky and Piskunow [4]. The theory by Weinberger [5] does not generalize results on convergence in shape obtained by Kolmogorov, Petrowsky and Piskunow [4, which shows that the width of the transition layer between the high and low parts of a spreading pulse remains bounded. This paper generalizes results on convergence in shape as defined in Theorem 5 or as defined by Weinberger.

Zinner et al. 6] have studied the existence of traveling wave solutions to spatialdiscretizations of Fisher's equation. The necessary and sufficient condition for the flame velocity agrees with the discrete KPP-theory above in the limit, when $k \rightarrow 0$.

\section{A DiscRete KPP-THEORY}

The first theorem gives sufficient conditions, such that $v(x, n k)$, defined by $(1.7)-$ (1.8), is bounded, continuous and strictly monotone.

Theorem 1. Assume that $F(u)$ is a continuous function on $[0,1]$, with a bounded and continuous derivative on $(0,1)$, and which satisfies the conditions (1.3)-(1.5). Let $v$ be the solution of (1.7)-(1.8).

Assume further that the time step $k$ and space step $h$ satisfy the inequality

$$
2 \lambda+k \kappa<1 \text {, where } \lambda=k / h^{2} \text { and } \kappa=\sup _{0<u<1}\left|F^{\prime}(u)\right| \text {. }
$$

Then we have for $n \geq 0$ :

(i)

$$
\begin{aligned}
& v(x, n k)=0, x \leq-n h, \\
& 0<v(x, n k)<1,-n h<x<(n+1) h, \\
& v(x, n k)=1, \quad(n+1) h \leq x .
\end{aligned}
$$

(ii) $v(x, n k)$ is a continuous function of $x$ for all $x$.

(iii) Let $\Delta>0$. Then

$$
v(x-\Delta, n k)<v(x, n k)<v(x+\Delta, n k),-n h<x<(n+1) h .
$$


Proof. Since the proof is elementary, we only prove (iii). From (1.7), (1.8) follows that the derivative

$$
w(x, n k)=\partial v(x, n k) / \partial x,
$$

exists for all $x \neq i h, \forall i$, and satisfies the difference equation

$$
\begin{gathered}
w(x,(n+1) k)=\left(1-2 \lambda+k F^{\prime}(v(x, n k)) w(x, n k)+\lambda w(x-h, n k)\right. \\
+\lambda w(x+h, n k),-\infty<x<\infty, x \neq i h, \forall i .
\end{gathered}
$$

The initial condition is

$$
w(x, 0)=1 / h, 0<x<h ; w(x, 0)=0, x<0, x>h .
$$

Since by $(2.1)$

$$
1-2 \lambda+k F^{\prime}(u)>0,0<u<1,
$$

it follows from (2.3) and (2.4),

$$
w(x, n k)>0,-n h<x<(n+1) h, x \neq i h,-n<i<n+1 .
$$

The strict monotonicty (2.2) follows from (2.5) and the mean value theorem.

The second theorem is a discrete counterpart to Theorem 10 in [4].

Theorem 2. Assume that the conditions in Theorem 1 are satisfied. Let $x$ be fixed. Then

$$
\lim _{n \rightarrow \infty} v(x-\beta n k, n k)=0
$$

if

$$
\beta k / h>\inf _{\alpha>0}\{\log [1-2 \lambda+k+\lambda(\exp (\alpha h)+\exp (-\alpha h))] /(\alpha h)\},
$$

where $\lambda=k / h^{2}$.

Proof. Introduce, for $\alpha>0, \beta>0$,

$$
w(x, n k)=v(x-\beta n k, n k) \exp (-\alpha x),-\infty<x<\infty,
$$

where $\alpha$ and $\beta$ are constants. Then $w$ satisfies

$$
\begin{aligned}
& w(x,(n+1) k)=(1-2 \lambda) \exp (-\alpha \beta k) w(x-\beta k, n k) \\
& \quad+\lambda \exp (\alpha(-h-\beta k)) w(x-\beta k-h, n k) \\
& \quad+\lambda \exp (\alpha(h-\beta k)) w(x-\beta k+h, n k) \\
& \quad+k \exp (-\alpha x) F\{w(x-\beta k, n k) \exp (\alpha(x-\beta k))\},-\infty<x<\infty,
\end{aligned}
$$

with

$$
w(x, 0)=v(x, 0) \exp (-\alpha x),-\infty<x<\infty .
$$

Lemma 1. Define $z(x, n k)$ by

$$
\begin{aligned}
& z(x,(n+1) k)=(1-2 \lambda) \exp (-\alpha \beta k) z(x-\beta k, n k) \\
& \quad+\lambda \exp (\alpha(-h-\beta k)) z(x-\beta k-h, n k) \\
& \quad+\lambda \exp (\alpha(h-\beta k)) z(x-\beta k+h, n k) \\
& \quad+k \exp (-\alpha \beta k) z(x-\beta k, n k),-\infty<x<\infty,
\end{aligned}
$$

where $z(x, 0)$ satisfies

$$
z(x, 0)=0, x<0 ; z(x, 0)=\exp (-\alpha x), x>0 .
$$


Then

$$
w(x, n k) \leq z(x, n k),-\infty<x<\infty, \forall n \geq 0 .
$$

Proof. The result follows by induction from (2.10) and the inequalities

$$
\begin{aligned}
& w(x, 0) \leq z(x, 0), \\
& F(u) \leq u, 0 \leq u \leq 1, \\
& 1-2 \lambda>0,
\end{aligned}
$$

which follow from (1.5), (2.1), (2.9) and (2.11).

Proof of Theorem 2 (cont.) We first choose $\beta$, such that (2.7) is satisfied. Then from (2.7) it follows that for some $\alpha>0$,

$$
\beta k / h>\log [1-2 \lambda+k+\lambda(\exp (\alpha h)+\exp (-\alpha h))] /(\alpha h) .
$$

From (2.8) and Lemma 1 it follows that (2.6) is true if $z(x, n k) \rightarrow 0, n \rightarrow \infty$, for fixed $x$ and $\alpha, \beta$, chosen as above.

Introduce the Fourier transform

$$
z_{f}(y, n k)=\int_{-\infty}^{+\infty} z(x, n k) \exp (-i y x) d x,-\infty<y<\infty
$$

$z_{f}(y, 0)$ exists since $\alpha>0$ in $(2.11)$.

We have from (2.10),

$$
z_{f}(y,(n+1) k)=A(y) z_{f}(y, n k),
$$

where

$$
A(y)=\exp (-\alpha \beta k-i \beta k y)[1-2 \lambda+k+\lambda \exp (-\alpha h-i h y)+\lambda \exp (\alpha h+i h y)] .
$$

Successive application of (2.12) gives

$$
z_{f}(y, n k)=\{A(y)\}^{n} z_{f}(y, 0) .
$$

Inversion of the Fourier transform gives

$$
z(x, n k)=\exp (-\alpha \beta n k)\left\{\sum_{j} c_{j}^{n} z(x-\beta n k+j h, 0)\right\},
$$

where

$$
c_{j}^{n}=(1 / 2 \pi) \int_{-\pi}^{\pi}(B(\theta))^{n} \exp (-i j \theta) d \theta
$$

and

$$
B(\theta)=1-2 \lambda+k+\lambda(\exp (-\alpha h-i \theta)+\exp (\alpha h+i \theta)) .
$$

(2.13) is proved by showing that $z(x, n k)$ defined in $(2.13)$ satisfies the difference equation (2.10).

We get from (2.11) and (2.13),

$$
z(x, n k)=\exp (-\alpha \beta n k) \sum_{j>-(x-\beta n k) / h} c_{j}^{n} \exp (-\alpha(x-\beta n k+j h)) .
$$

Let $x$ be fixed. Then from (2.14)

$$
|z(x, n k)| \leq \exp (-\alpha \beta n k)\left\{\sup _{|\theta| \leq \pi}|B(\theta)|\right\}^{n} \sum_{j=0}^{\infty} \exp (-\alpha j h),
$$


and

$$
\begin{aligned}
& \sup _{|\theta| \leq \pi}|B(\theta)|=\sup _{|\theta| \leq \pi}\left\{(1-2 \lambda+k)^{2}\right. \\
& +2(1-2 \lambda+k) \lambda(\exp (\alpha h)+\exp (-\alpha h)) \cos (\theta) \\
& +\lambda^{2}(\exp (\alpha h)+\exp (-\alpha h))^{2} \cos ^{2}(\theta) \\
& \left.+\lambda^{2}(\exp (\alpha h)-\exp (-\alpha h))^{2}\left(1-\cos ^{2}(\theta)\right)\right\}^{1 / 2} \\
& =\sup _{|\theta| \leq \pi}\left\{(1-2 \lambda+k)^{2}\right. \\
& +2(1-2 \lambda+k) \lambda(\exp (\alpha h)+\exp (-\alpha h)) \cos (\theta) \\
& \left.+\lambda^{2}(\exp (\alpha h)-\exp (-\alpha h))^{2}+4 \lambda^{2} \cos ^{2}(\theta)\right\}^{1 / 2} \\
& =\left\{(1-2 \lambda+k)^{2}+2(1-2 \lambda+k) \lambda(\exp (\alpha h)+\exp (-\alpha h))\right. \\
& \left.+\lambda^{2}(\exp (\alpha h)-\exp (-\alpha h))^{2}+4 \lambda^{2}\right\}^{1 / 2},
\end{aligned}
$$

which finally gives

$$
\sup _{|\theta| \leq \pi}|B(\theta)|=1-2 \lambda+k+\lambda(\exp (\alpha h)+\exp (-\alpha h))=B(0) .
$$

We have for fixed $x$, from (2.15) and (2.16),

$$
\lim _{n \rightarrow \infty} z(x, n k)=0
$$

if

$$
\exp (-\alpha \beta k)\{1-2 \lambda+k+\lambda(\exp (\alpha h)+\exp (-\alpha h))\}<1,
$$

or equivalently,

$$
\beta k / h>\log [1-2 \lambda+k+\lambda(\exp (\alpha h)+\exp (-\alpha h))] /(\alpha h),
$$

which is true according to our choice of $\alpha$ and $\beta$ above.

Theorem 3 is a discrete version of Theorem 11 in [4].

Theorem 3. Assume that the conditions in Theorem 1 are satisfied. Assume further that

$$
3 k / h^{2}+k \kappa<1,
$$

where $\kappa$ is defined in (2.1). Let $\varphi^{n}$ be defined by (1.9). Then $v\left(\varphi^{n}+i h, n k\right)$ is a decreasing function of $n$ for all fixed $i>0$ and an increasing function of $n$ for all fixed $i<0$.

Proof. Introduce

$$
w(x, n k)=v(x, n k)-v(x+C,(n+1) k),
$$

where $C$ is a fixed but arbitrary constant. We first observe that $w(x, n k)$ satisfies the difference equation

$$
\begin{aligned}
w(x,(n+1) k)= & (1-2 \lambda) w(x, n k)+\lambda w(x-h, n k)+\lambda w(x+h, n k) \\
& +k K(x, n k) w(x, n k),
\end{aligned}
$$

where

$$
K(x, n k)=F^{\prime}(\eta)
$$

and $\eta$ is a number between $v(x, n k)$ and $v(x+C,(n+1) k)$. We first study $w(x, n k)$. 
Lemma 2. Introduce

$$
x_{i}=i h+\Delta, \forall i \in Z,
$$

where $\Delta$ is a fixed but arbitrary constant. Assume that (2.17) is satisfied. Assume that, for some $i$,

$$
w\left(x_{i-1}, n k\right) \leq 0, w\left(x_{i}, n k\right) \leq 0, w\left(x_{i+1}, n k\right) \geq 0, \text { and } w\left(x_{i+2}, n k\right) \geq 0 .
$$

Then

$$
w\left(x_{i+1},(n+1) k\right) \geq 0 \text { if } w\left(x_{i},(n+1) k\right) \geq 0
$$

and

$$
w\left(x_{i+1},(n+1) k\right)>0 \text { if } w\left(x_{i},(n+1) k\right)>0 .
$$

Proof. Assume that $w\left(x_{i+1},(n+1) k\right)<0$ and $w\left(x_{i},(n+1) k\right) \geq 0$. Define

$$
a=w\left(x_{i-1}, n k\right), b=w\left(x_{i}, n k\right), d=w\left(x_{i+1}, n k\right) \text { and } e=w\left(x_{i+2}, n k\right) .
$$

Then

$$
w\left(x_{i},(n+1) k\right)=\lambda a+(1-2 \lambda) b+\lambda d+k K\left(x_{i}, n k\right) b \geq 0
$$

and

$$
-w\left(x_{i+1},(n+1) k\right)=-\left(\lambda b+(1-2 \lambda) d+\lambda e+k K\left(x_{i+1}, n k\right) d\right)>0,
$$

by assumption. Addition of these inequalities gives

$$
\lambda a+\left(1-3 \lambda+k K\left(x_{i}, n k\right)\right) b+\left(3 \lambda-1-k K\left(x_{i+1}, n k\right)\right) d-\lambda e>0 .
$$

From the assumptions (2.17) and (2.19) follows that $a \leq 0, b \leq 0, d \geq 0$ and $e \geq 0$. Furthermore,

$$
\begin{aligned}
& \left(1-3 \lambda+k K\left(x_{i}, n k\right)\right) \geq 1-3 \lambda-k \kappa>0, \\
& \left(3 \lambda-1-k K\left(x_{i+1}, n k\right)\right) \leq 3 \lambda-1+k \kappa=-(1-3 \lambda-k \kappa)<0 .
\end{aligned}
$$

This contradicts (2.22) and we conclude that (2.20) holds. Similarly, we prove that (2.21) is valid.

Let $i_{0}$ denote the largest integer for which

$$
v\left(x_{i}+C, k\right)=v\left(x_{i}, 0\right)=0, \forall i \leq i_{0},
$$

and $i_{1}$ the smallest number for which

$$
v\left(x_{i}+C, k\right)=v\left(x_{i}, 0\right)=1, \forall i \geq i_{1} .
$$

Then we derive from $(2.17),(2.18),(2.23)$ and (2.24) that

$$
w\left(x_{i}, n k\right)=0, i \leq i_{0}-n, i \geq i_{1}+n, \forall n \geq 0 .
$$

Lemma 3. There exist three cases:

Case 1.

$$
w\left(x_{i}, n k\right) \geq 0, i_{0}-n<i<i_{1}+n, \forall n \geq 0 .
$$

Case 2.

$$
w\left(x_{i}, n k\right)<0, i_{0}-n<i<i_{1}+n, \forall n \geq 0 .
$$


Case 3. There exist numbers $i(n)$ such that

$$
\begin{aligned}
& i(0)-n \leq i(n) \leq i(0)+n, \forall n \geq 0, \\
& w\left(x_{i}, n k\right)<0, i_{0}-n<i \leq i(n), \forall n \geq 0, \\
& w\left(x_{i}, n k\right) \geq 0, i(n)<i<i_{1}+n, \forall n \geq 0 .
\end{aligned}
$$

Proof. We consider first the case when $n=0$. If

$$
v\left(x_{i_{0}+1}, 0\right) \geq v\left(x_{i_{0}+1}+C, k\right),
$$

then

$$
v\left(x_{i_{0}+1}, 0\right)>0 \text { and } v\left(x_{i_{0}+2}, 0\right)=1 \text {, }
$$

by the definition of $v\left(x_{i}, 0\right)$ in $(1.8)$ and $i_{0}$ in (2.23). Case 1 is therefore valid. If

$$
v\left(x_{i_{0}+1}, 0\right)<v\left(x_{i_{0}+1}+C, k\right),
$$

then

$$
v\left(x_{i_{0}+2}, 0\right) \geq v\left(x_{i_{0}+2}+C, k\right) \text { or } v\left(x_{i_{0}+2}, 0\right)<v\left(x_{i_{0}+2}+C, k\right) .
$$

In the first case we have $i(0)=i_{0}+1$ and case 3 is valid. In the second case, i.e., if

$$
v\left(x_{i_{0}+2}, 0\right)<v\left(x_{i_{0}+2}+C, k\right),
$$

then

$$
v\left(x_{i_{0}+3}, 0\right) \geq v\left(x_{i_{0}+3}+C, k\right) \text { or } v\left(x_{i_{0}+3}, 0\right)<v\left(x_{i_{0}+3}+C, k\right) .
$$

In the first case we have $i(0)=i_{0}+2$. The proof is continued in a finite number of steps and proves that Case 1, Case 2 or Case 3 are the only possible cases for $n=0$.

Case 1 implies, for $n=0$,

$$
w\left(x_{i}, 0\right) \geq 0, i_{0}<i<i_{1} .
$$

(2.17), (2.18), (2.23) and (2.24) give by induction,

$$
w\left(x_{i}, n k\right) \geq 0, i_{0}-n<i<i_{1}+n, \forall n \geq 0 .
$$

Case 2 implies, for $n=0$,

$$
w\left(x_{i}, 0\right)<0, i_{0}<i<i_{1}
$$

and, in general,

$$
w\left(x_{i}, n k\right)<0, i_{0}-n<i<i_{1}+n, \forall n \geq 0 .
$$

Case 3 implies for $n=0$ that there exists a number $i(0)$, such that

$$
\begin{aligned}
& w\left(x_{i}, 0\right)<0, i_{0}<i \leq i(0), \\
& w\left(x_{i}, 0\right) \geq 0, i(0)<i<i_{1} .
\end{aligned}
$$

(2.17), (2.18), (2.23) and (2.24) then give

$$
\begin{aligned}
& w\left(x_{i}, k\right)=0, i \leq i_{0}-1, i \geq i_{1}+1, \\
& w\left(x_{i}, k\right)<0, i_{0}-1<i \leq i(0)-1, \\
& w\left(x_{i}, k\right) \geq 0, i(0)+1<i<i_{1}+1 .
\end{aligned}
$$

Lemma 2 gives

$$
w\left(x_{i(0)+1}, k\right) \geq 0 \text { if } w\left(x_{i(0)}, k\right) \geq 0
$$

and

$$
w\left(x_{i(0)}, k\right)<0 \text { if } w\left(x_{i(0)+1}, k\right)<0 .
$$


We therefore conclude that there exists a number $i(1)$, which is equal to $i(0)-1, i(0)$ or $i(0)+1$, such that

$$
\begin{aligned}
& w\left(x_{i}, k\right)<0, \quad i_{0}-1<i \leq i(1), \\
& w\left(x_{i}, k\right) \geq 0, \quad i(1)<i \leq i_{1}+1 .
\end{aligned}
$$

By induction it follows that there exists a number $i(n)$, such that

$$
\begin{aligned}
& i(0)-n \leq i(n) \leq i(0)+n, \forall n \geq 0, \\
& w\left(x_{i}, n k\right)<0, i_{0}-n<i \leq i(n), \forall n \geq 0, \\
& w\left(x_{i}, n k\right) \geq 0, i(n)<i<i_{1}+n, \forall n \geq 0,
\end{aligned}
$$

i.e., Lemma 3 is proved.

Proof of Theorem 3 (cont.) Let $v_{0}$ be a fixed, but arbitrary constant, such that $0<v_{0}<1$.

In order to prove Theorem 3 , we choose a number $X_{n}$, depending on $n$ and $v_{0}$, such that

$$
v\left(X_{n}, n k\right)=v_{0} .
$$

This is possible since $v(x, n k)$ is continuous and strictly monotone for $0<v(x, n k)<$ 1 , by Theorem 1 . We then choose $C_{n}$ such that

$$
v\left(X_{n}+C_{n},(n+1) k\right)=v\left(X_{n}, n k\right)=v_{0} .
$$

Then

$$
w\left(X_{n}, n k\right)=0,
$$

which implies that case 2 in Lemma 3 is not valid, since $0<v_{0}<1$. It then follows from (2.25) or (2.28) that

$$
w\left(X_{n}+i h, n k\right) \geq 0, i \geq 1,
$$

which, with (2.29), implies

$$
v\left(X_{n}+i h, n k\right)-v\left(X_{n}, n k\right) \geq v\left(X_{n}+i h+C_{n},(n+1) k\right)-v\left(X_{n}+C_{n},(n+1) k\right),
$$

which proves the case $i \geq 1$.

The case $i \leq-1$ is proved by replacing Cases $1-3$ in Lemma 3, by

Case 1'.

$$
w\left(x_{i}, n k\right)>0, i_{0}-n<i<i_{1}+n, \forall n \geq 0 .
$$

Case 2'.

$$
w\left(x_{i}, n k\right) \leq 0, i_{0}-n<i<i_{1}+n, \forall n \geq 0 .
$$

Case $3^{\prime}$. There exists a number $i(n)$, such that

$$
\begin{aligned}
& i(0)-n \leq i(n) \leq i(0)+n, \forall n \geq 0 \\
& w\left(x_{i}, n k\right) \leq 0, i_{0}-n<i \leq i(n), \forall n \geq 0 \\
& w\left(x_{i}, n k\right)>0, i(n)<i<i_{1}+n, \forall n \geq 0 .
\end{aligned}
$$

The proof of Cases $1^{\prime}-3^{\prime}$ is similar to the proof of Cases 1-3 in Lemma 3. 
Theorem 4. Assume that the conditions in Theorem 1 are satisfied. Define the function $v^{*}(x, n k)$ by

$$
v^{*}(x, n k)=v\left(x+\varphi^{n}, n k\right),
$$

where $\varphi^{n}$ is defined by (1.9). Then

$$
v^{*}(0, n k)=v\left(\varphi^{n}, n k\right)=c,
$$

and

$$
\lim _{n \rightarrow \infty} v^{*}(i h, n k)=v^{*}(i h), \forall i \in Z
$$

exists.

Proof. From Theorem 3 follows that $v^{*}(i h, n k), i \geq 1$, is a nonincreasing function of $n$ and $v^{*}(i h, n k), i \leq-1$, is a nondecreasing function of $n$.

Since $v^{*}(i h, n k)$ is bounded below by zero and above by one, it follows that

$$
\lim _{n \rightarrow \infty} v^{*}(i h, n k)=v^{*}(i h), \forall i,
$$

exists.

Theorem 5 is a discrete analogue to Theorem 14 in [4].

Theorem 5. The function

$$
v_{m}(i h, n k)=v\left(i h+\varphi^{m},(n+m) k\right), \forall i, \forall n \geq 0, \forall m \geq 0,
$$

converges as $m \rightarrow \infty$, towards a function $V(i h, n k)$, which satisfies the difference equation

$$
\begin{aligned}
& V(i h,(n+1) k)=(1-2 \lambda) V(i h, n k)+\lambda V(i h-h, n k))+\lambda V(i h+h, n k) \\
& \quad+k F(V(i h, n k)), \forall i, \forall n \geq 0,
\end{aligned}
$$

with initial data

$$
V(i h, 0)=v^{*}(i h), \forall i,
$$

where $v^{*}(i h)$ is defined in Theorem 4.

Proof. From Theorem 4 follows that

$$
v_{m}(i h, 0)=v\left(i h+\varphi^{m}, m k\right)=v^{*}(i h, m k) \rightarrow v^{*}(i h), m \rightarrow \infty, \forall i .
$$

Then

From (1.7) follows:

$$
V(i h, 0)=v^{*}(i h), \forall i
$$

$$
\begin{aligned}
& v_{m}(i h, k)=v\left(i h+\varphi^{m},(m+1) k\right)=(1-2 \lambda) v\left(i h+\varphi^{m}, m k\right) \\
& \quad+\lambda v\left(i h+\varphi^{m}-h, m k\right)+\lambda v\left(i h+\varphi^{m}+h, m k\right) \\
& \quad+k F\left(v\left(i h+\varphi^{m}, m k\right)\right), \forall i, \forall m \geq 0,
\end{aligned}
$$

which converges, as $m \rightarrow \infty$, towards a solution $V(i h, k)$ to the difference equation (2.30). By induction it follows that $v_{m}(i h, n k)$ converges towards a function $V(i h, n k)$, which satisfies (2.30).

In order to study the properties of $V$, defined in Theorem 5 , we first derive an upper estimate for $\varphi^{n}-\varphi^{n+1}$.

Lemma 4. Assume that $h$ and $k$ satisfy the condition (2.1) in Theorem 1 . Then

$$
\varphi^{n}-\varphi^{n+1} \leq h, \forall n \geq 0 .
$$


Proof. Introduce

$$
z(x, n k)=v(x-h,(n+1) k)-v(x, n k),-\infty<x<\infty, \forall n \geq 0 .
$$

Then, from (1.7),

$$
\begin{aligned}
z(x,(n+1) k) & =(1-2 \lambda) z(x, n k)+\lambda z(x-h, n k)+\lambda z(x+h, n k) \\
& +k F^{\prime}\left(v^{n}\right) z(x, n k), \forall n \geq 0,
\end{aligned}
$$

where $v^{n}$ is a number between $v(x-h,(n+1) k)$ and $v(x, n k)$.

Using $n=0$ in (2.31) gives

$$
z(x, 0)=v(x-h, k)-v(x, 0) .
$$

(1.7) and (1.8) give, for $0<x<h$,

$$
z(x, 0)=\lambda v(x, 0)-v(x, 0)<0,0<x<h .
$$

Also,

$$
z(x, 0) \leq 0, x \leq 0 \text { and } x \geq h .
$$

From (2.1) and (2.32) it follows by induction that

$$
v(x-h,(n+1) k) \leq v(x, n k), \forall n \geq 0,
$$

which implies

$$
\varphi^{n+1}-\varphi^{n} \geq-h, \forall n \geq 0 .
$$

Lemma 5. $V(i h, n k)$ has the properties

$$
\begin{aligned}
& \text { i) } \quad V(i h, n k) \leq V((i+1) h, n k), \forall i, \forall n \geq 0, \\
& \text { ii) } 0<V(i h, n k)<1, \quad \forall i, \forall n \geq 0, \\
& \text { iii) } \quad \lim _{i \rightarrow \infty} V(i h, n k)=1, \forall n \geq 0, \\
& \text { iv) } \quad \lim _{i \rightarrow-\infty} V(i h, n k)=0, \forall n \geq 0 .
\end{aligned}
$$

Proof. i) $V$ is monotone since $v$ is monotone.

ii) (2.34) follows from Theorem 3.

iii) Introduce

$$
\lim _{i \rightarrow \infty} V(i h, 0)=\alpha
$$

which exists since $V$ is monotone by i) and bounded by ii).

Then $\alpha>0$ since $V(0,0)=c$ and $0<\mathrm{c}<1$.

Assume that $\alpha<1$. Then

$$
\begin{aligned}
& V(i h, k)=\lim _{n \rightarrow \infty} v\left(i h+\varphi^{n},(n+1) k\right) \\
& \quad=\lim _{n \rightarrow \infty} v\left(i h+\varphi^{n}-\varphi^{n+1}+\varphi^{n+1},(n+1) k\right) \\
& \quad \leq \lim _{n \rightarrow \infty} v\left(i h+h+\varphi^{n+1},(n+1) k\right)=V(i h+h, 0) \leq \alpha<1,
\end{aligned}
$$

by Lemma 4 . From (2.30)

$$
\begin{aligned}
V(i h, k) & =(1-2 \lambda) V(i h, 0)+\lambda V(i h-h, 0) \\
& +\lambda V(i h+h, 0)+k F(V(i h, 0)), \forall i,
\end{aligned}
$$

which implies

$$
\lim _{i \rightarrow \infty} V(i h, k)=(1-2 \lambda) \alpha+2 \lambda \alpha+k F(\alpha)=\alpha+k F(\alpha)>\alpha,
$$

if $0<\alpha<1$. 
The contradiction implies that

$$
\lim _{i \rightarrow \infty} V(i h, 0)=1 .
$$

Similarly, we prove by induction that

$$
\lim _{i \rightarrow \infty} V(i h, n k)=1, \forall n \geq 0 .
$$

iv) Similar arguments can be used to prove

$$
\lim _{i \rightarrow-\infty} V(i h, n k)=0, \forall n \geq 0 .
$$

Numerical experiments suggest that $\varphi^{n}-\varphi^{n+1}$ fluctuates as $n$ increases. Theorem 6 shows that $\lim _{n \rightarrow \infty} \varphi^{n} / n$ exists.

Theorem 6. Assume that the conditions in Theorems 1 and 3 are satisfied. Then

$$
\lim _{n \rightarrow \infty} \varphi^{n} / n=\gamma h \text {. }
$$

Proof. The proofs relies on the following lemmas.

Lemma 6. Assume that the conditions in Theorems 1 and 3 are satisfied. Let $c$ be a fixed, but arbitrary constant defined in (1.9). Then, there exists for every $m \geq 0$ a unique number $j_{m}$, such that

$$
V\left(\left(j_{m}-1\right) h, m k\right) \leq c<V\left(j_{m} h, m k\right), \forall m \geq 0 .
$$

Proof. The result follows directly from Lemma 5 .

Lemma 7. Assume that the conditions of Lemma 6 are satisfied. Let $j_{m}$ be as in Lemma 6. Then

$$
\begin{gathered}
\limsup _{n \rightarrow \infty}\left(\varphi^{n}-\varphi^{n+m}\right) \leq-\left(j_{m}-1-j_{0}\right) h, \forall m \geq 0, \\
\liminf _{n \rightarrow \infty}\left(\varphi^{n}-\varphi^{n+m}\right) \geq-\left(j_{m}+1-j_{0}\right) h, \forall m \geq 0 .
\end{gathered}
$$

Proof. We first prove (2.38).

From the definition of $V$ in Theorem 5 follows:

$$
\begin{aligned}
& V\left(\left(j_{m}-1\right) h, m k\right)=\lim _{n \rightarrow \infty} v\left(\left(j_{m}-1\right) h+\varphi^{n},(m+n) k\right) \\
& \quad=\lim _{n \rightarrow \infty} v\left(\left(j_{m}-1\right) h+\varphi^{n}-\varphi^{n+m}+\varphi^{n+m},(m+n) k\right) \leq c,
\end{aligned}
$$

according to (2.37). We then claim that

$$
\left(j_{m}-1\right) h+\limsup _{n \rightarrow \infty}\left(\varphi^{n}-\varphi^{n+m}\right) \leq j_{0} h, \forall m \geq 0 .
$$

If the claim is not true, we can find some $m \geq 0$, such that

$$
\left(j_{m}-1\right) h+\limsup _{n \rightarrow \infty}\left(\varphi^{n}-\varphi^{n+m}\right)>j_{0} h .
$$

Then there exists a subsequence $\{n(i)\}_{i \geq 1}$, such that

$$
\left(j_{m}-1\right) h+\left(\varphi^{n(i)}-\varphi^{n(i)+m}\right)>j_{0} h, \forall i \geq 1
$$

and

$$
\left(j_{m}-1\right) h+\lim _{i \rightarrow \infty}\left(\varphi^{n(i)}-\varphi^{n(i)+m}\right)>j_{0} h .
$$


(2.40) and (2.42) give

$$
\begin{aligned}
& V\left(\left(j_{m}-1\right) h, m k\right) \geq \lim _{i \rightarrow \infty} v\left(j_{0} h+\varphi^{n(i)+m},(n(i)+m) k\right) \\
& \quad=V\left(j_{0} h, 0\right)>c,
\end{aligned}
$$

from the definition of $j_{0}$ in $(2.37)$.

The contradiction between (2.40) and (2.43) shows that the claim is true, i.e.,

$$
\left(j_{m}-1\right) h+\limsup _{n \rightarrow \infty}\left(\varphi^{n}-\varphi^{n+m}\right) \leq j_{0} h, \forall m \geq 0 .
$$

(2.41) is equivalent to (2.38). Similarly, we prove that

$$
j_{m} h+\liminf _{n \rightarrow \infty}\left(\varphi^{n}-\varphi^{n+m}\right) \geq\left(j_{0}-1\right) h, \forall m \geq 0 .
$$

Proof of Theorem 6 . Let $m \geq 1$ be a fixed but arbitrary number. Then for every $\varepsilon>0$, there exists a number $M \geq 0$, such that

$$
\varphi^{n}-\varphi^{n-m} \leq \limsup _{n \rightarrow \infty}\left(\varphi^{n}-\varphi^{n-m}\right)+\varepsilon, n-m \geq M .
$$

Write

$$
\begin{aligned}
\varphi^{n} & =\left(\varphi^{n}-\varphi^{n-m}\right)+\left(\varphi^{n-m}-\varphi^{n-2 m}\right)+\cdots \\
& +\left(\varphi^{n-(L-1) m}-\varphi^{n-L m}\right)+\varphi^{n-L m},
\end{aligned}
$$

where $L$ is the largest number that satisfies $n-L m \geq M$.

Then, we derive from (2.44),

$$
\begin{aligned}
& \varphi^{n} \leq L\left\{\limsup _{n \rightarrow \infty}\left(\varphi^{n}-\varphi^{n-m}\right)+\varepsilon\right\}+C_{M}, \\
& C_{M}=\max \left\{\varphi^{M}, \varphi^{M+1}, \ldots, \varphi^{M+m-1}\right\},
\end{aligned}
$$

so that

$$
\varphi^{n} \leq((n-M) / m)\left\{\limsup _{n \rightarrow \infty}\left(\varphi^{n}-\varphi^{n-m}\right)+\varepsilon\right\}+C_{M},
$$

and

$$
\varphi^{n} / n \leq\left((n-M) /(m n)\left\{\limsup _{n \rightarrow \infty}\left(\varphi^{n}-\varphi^{n-m}\right)+\varepsilon\right\}+C_{M} / n,\right.
$$

which gives

$$
\limsup _{n \rightarrow \infty} \varphi^{n} / n \leq(1 / m)\left\{\limsup _{n \rightarrow \infty}\left(\varphi^{n}-\varphi^{n-m}\right)+\varepsilon\right\} .
$$

Since $\varepsilon$ is arbitrary, we derive

$$
\limsup _{n \rightarrow \infty} \varphi^{n} / n \leq(1 / m)\left\{\limsup _{n \rightarrow \infty}\left(\varphi^{n}-\varphi^{n-m}\right)\right\}, \forall m \geq 1 .
$$

Similarly, we derive

$$
\liminf _{n \rightarrow \infty} \varphi^{n} / n \geq(1 / m)\left\{\liminf _{n \rightarrow \infty}\left(\varphi^{n}-\varphi^{n-m}\right)\right\}, \forall m \geq 1 .
$$

Since

$$
\begin{aligned}
& \liminf _{n \rightarrow \infty}\left(\varphi^{n}-\varphi^{n-m}\right)=-\limsup _{n \rightarrow \infty}\left(\varphi^{n-m}-\varphi^{n}\right) \\
& \quad=-\limsup _{n \rightarrow \infty}\left(\varphi^{n}-\varphi^{n+m}\right), \\
& \limsup _{n \rightarrow \infty}\left(\varphi^{n}-\varphi^{n-m}\right)=-\liminf _{n \rightarrow \infty}\left(\varphi^{n-m}-\varphi^{n}\right) \\
& \quad=-\liminf _{n \rightarrow \infty}\left(\varphi^{n}-\varphi^{n+m}\right),
\end{aligned}
$$


we derive from Lemma $7,(2.45)$ and $(2.46)$

$$
\left(j_{m}-1-j_{0}\right) h / m \leq \liminf _{n \rightarrow \infty} \varphi^{n} / n \leq \limsup _{n \rightarrow \infty} \varphi^{n} / n \leq\left(j_{m}+1-j_{0}\right) h / m .
$$

Since the difference between the upper and lower estimate in $(2.47)$ differ by $2 h / m$, which converges to zero as $m \rightarrow \infty$, it follows that

$$
\liminf _{n \rightarrow \infty} \varphi^{n} / n=\limsup _{n \rightarrow \infty} \varphi^{n} / n,
$$

i.e., $\lim _{n \rightarrow \infty} \varphi^{n} / n$ exists, which proves Theorem 6 .

Theorem 7. Assume that $\gamma$, defined in Theorem 6, is a rational number. Let

$$
\gamma=P / Q,
$$

where $P$ and $Q>0$ are integers with no common factor. Then

$$
\lim _{L \rightarrow \infty} V((i+L P) h,(j+L Q) k)=W(i h-j h P / Q),
$$

exists for $\forall i, \forall j \geq 0$, where $W$ is uniquely defined and satisfies the difference equation

$$
\begin{aligned}
& W(i h-(j+1) h P / Q)=(1-2 \lambda) W(i h-j h P / Q)+\lambda W(i h-h-j h P / Q) \\
& +\lambda W(i h+h-j h P / Q)+k F(W(i h-j h P / Q)), \quad \forall i, \quad \forall j \geq 0 .
\end{aligned}
$$

It follows from Lemma 5 and the proof of Theorem 7 that

$$
\begin{aligned}
& \lim _{i \rightarrow-\infty} W(i h-j h P / Q)=0, \\
& \lim _{i \rightarrow \infty} W(i h-j h P / Q)=1 .
\end{aligned}
$$

Proof. The proofs relies on the following lemmas.

Lemma 8. Introduce

$$
\alpha_{L}^{n}=\varphi^{n}-\varphi^{n+Q L}+L P h, \forall n \geq 0, \forall L \geq 1 .
$$

Define

$$
\begin{gathered}
\alpha_{L}^{+}=\limsup _{n \rightarrow \infty} \alpha_{L}^{n}, \forall n \geq 0, \forall L \geq 1, \\
\alpha_{L}^{-}=\liminf _{n \rightarrow \infty} \alpha_{L}^{n}, \quad \forall n \geq 0, \forall L \geq 1 .
\end{gathered}
$$

Then

$$
-2 h \leq \alpha_{L}^{-} \leq 0 \leq \alpha_{L}^{+} \leq 2 h, \forall L \geq 1 .
$$

Proof. Assume that $\alpha_{L}^{-}>0$. Then there exists a number $M_{L}$, such that

$$
\alpha_{L}^{n}>\left(\alpha_{L}^{-}\right) / 2, n \geq M_{L}
$$

or

$$
\varphi^{n+Q L}-\varphi^{n}<L P h-\left(\alpha_{L}^{-}\right) / 2, n \geq M_{L}
$$

Summation gives:

$$
\varphi^{n+m Q L}-\varphi^{n}<m L P h-m\left(\alpha_{L}^{-}\right) / 2, n \geq M_{L}
$$

and

$$
\begin{aligned}
& \varphi^{n+m Q L} /(n+m Q L)-\varphi^{n} /(n+m Q L) \\
& \quad<m L P h /(n+m Q L)-m\left(\left(\alpha_{L}^{-}\right) / 2\right) /(n+m Q L), n \geq M_{L} .
\end{aligned}
$$

Let $m \rightarrow \infty$. Then it follows that

$$
(P / Q) h \leq(P / Q) h-\left(\alpha_{L}^{-}\right) /(2 Q L),
$$


which is a contradiction, since $Q>0$ and $\left(\alpha_{L}^{-}\right)>0$ by assumptions. Similarly, we prove that $\alpha_{L}^{+} \geq 0$.

Assume that $\alpha_{L}^{-}<-2 h$. Then there exists two subsequences $\left\{\varphi^{m(i)}\right\}, \forall i \geq 1$ and $\left\{\varphi^{n(i)}\right\}, \forall i \geq 1$, such that

$$
\begin{aligned}
& \alpha_{L}^{m(i)}<-2 h, \forall i \geq 1, \\
& \alpha_{L}^{m(i)} \rightarrow \alpha_{L}^{-}, i \rightarrow \infty, \\
& \alpha_{L}^{n(i)}>-h, \forall i \geq 1, \\
& \alpha_{L}^{n(i)} \rightarrow\left(\alpha_{L}^{+}\right) \geq 0, i \rightarrow \infty .
\end{aligned}
$$

Lemma 6 states that

$$
V\left(\left(j_{m}-1\right) h, m k\right) \leq c<V\left(j_{m} h, m k\right), \forall m \geq 0 .
$$

Then, we derive

$$
\begin{aligned}
& V\left(\left(j_{m}+1\right) h+L P h,(m+L Q) k\right) \\
& \quad=\lim _{i \rightarrow \infty} v\left(\left(j_{m}+1\right) h+L P h+\varphi^{m(i)},(m(i)+m+L Q) k\right) \\
& \quad=\lim _{i \rightarrow \infty} v\left(\left(j_{m}+1\right) h+\left(L P h+\varphi^{m(i)}-\varphi^{m(i)+L Q}\right)+\varphi^{m(i)+Q L},(m(i)+m+L Q) k\right) \\
& \quad \leq \lim _{i \rightarrow \infty} v\left(\left(j_{m}+1\right) h-2 h+\varphi^{m(i)+Q L},(m(i)+m+L Q) k\right) \\
& \quad=V\left(\left(j_{m}-1\right) h, m k\right) \leq c,
\end{aligned}
$$

by $(2.50)$. We also have

$$
\begin{aligned}
& V\left(\left(j_{m}+1\right) h+L P h,(m+L Q) k\right) \\
& \quad=\lim _{i \rightarrow \infty} v\left(\left(j_{m}+1\right) h+L P h+\varphi^{n(i)},(n(i)+m+L Q) k\right) \\
& \quad=\lim _{i \rightarrow \infty} v\left(\left(j_{m}+1\right) h+\left(L P h+\varphi^{n(i)}-\varphi^{n(i)+L Q}\right)+\varphi^{n(i)+L Q},(n(i)+m+L Q) k\right) \\
& \quad \geq \lim _{i \rightarrow \infty} v\left(j_{m} h+\varphi^{n(i)+L Q},(n(i)+m+L Q) k\right)=V\left(j_{m} h, m k\right)>c
\end{aligned}
$$

by (2.50). The contradiction proves that $\alpha_{L}^{-} \geq-2 h$. Similarly, we prove that $\alpha_{L}^{+} \leq 2 h$.

Lemma 9. Assume that

$$
\alpha_{1}^{-}<0<\alpha_{1}^{+}
$$

Then

$$
V((i+P) h,(j+Q) k)=V(i h, j k), \forall i, \forall j \geq 0,
$$

and $W$ exists by (2.48).

Proof. If (2.51) is satisfied, it follows that there exists two subsequences $\left\{\varphi^{m(L)}\right\}, \forall L \geq 1$ and $\left\{\varphi^{n(L)}\right\}, \forall L \geq 1$, such that

$$
\begin{aligned}
& \alpha_{1}^{m(L)}<0, \forall L \geq 1, \\
& \alpha_{1}^{m(L)} \rightarrow \alpha_{1}^{-}<0, L \rightarrow \infty, \\
& \alpha_{1}^{n(L)}>0, \forall L \geq 1, \\
& \alpha_{1}^{n(L)} \rightarrow \alpha_{1}^{+}>0, L \rightarrow \infty .
\end{aligned}
$$


Then

$$
\begin{aligned}
& V((i+P) h,(j+Q) k)=\lim _{L \rightarrow \infty} v\left((i+P) h+\varphi^{m(L)},(m(L)+j+Q) k\right) \\
& \quad=\lim _{L \rightarrow \infty} v\left(i h+\left(P h+\varphi^{m(L)}-\varphi^{m(L)+Q}\right)+\varphi^{m(L)+Q},(m(L)+j+Q) k\right) \\
& \quad \leq \lim _{L \rightarrow \infty} v\left(i h+\varphi^{m(L)+Q},(m(L)+j+Q) k\right)=V(i h, j k) .
\end{aligned}
$$

Similarly, we prove using $\left\{\varphi^{n(L)}\right\}, \forall L \geq 1$, that

$$
V((i+P) h,(j+Q) k) \geq V(i h, j k) .
$$

(2.53) and (2.54) imply

$$
V((i+P) h,(j+Q) k)=V(i h, j k), \forall i, \forall j \geq 0 .
$$

It then follows from the definition of $W$ in (2.48) that

$$
W(i h-j h P / Q)=V(i h, j k), \forall i, \forall j \geq 0 .
$$

Proof of Theorem 7. We have from Lemma 8 that

$$
\alpha_{1}^{-} \leq 0 \leq \alpha_{1}^{+} .
$$

We next consider the case

$$
\alpha_{1}^{-} \leq 0=\alpha_{1}^{+} .
$$

If there exists two subsequences, such that

$$
\begin{aligned}
& \alpha_{1}^{m(L)} \leq 0, \forall L \geq 1, \\
& \alpha_{1}^{m(L)} \rightarrow \alpha_{1}^{-} \leq 0, L \rightarrow \infty, \\
& \alpha_{1}^{n(L)} \geq 0, \forall L \geq 1, \\
& \alpha_{1}^{n(L)} \rightarrow \alpha_{1}^{+}=0, L \rightarrow \infty,
\end{aligned}
$$

then it follows, as in Lemma 9 , that

$$
V((i+P) h,(j+Q) k)=V(i h, j k), \forall i, \forall j \geq 0 .
$$

It is therefore sufficient to study the case, where

$$
\alpha_{1}^{-} \leq 0=\alpha_{1}^{+} \text {and } \alpha_{1}^{m} \leq 0, \forall m \geq 1 .
$$

Lemma 10. Assume that

$$
\begin{gathered}
\alpha_{1}^{-} \leq 0=\alpha_{1}^{+} \\
\alpha_{1}^{m}=P h+\varphi^{m}-\varphi^{m+Q} \leq 0, \forall m \geq 1 .
\end{gathered}
$$

The sequence

$$
\{V((i+L P) h,(j+L Q) k)\}, \forall L \geq 1,
$$

is then nonincreasing, and

$$
\lim _{L \rightarrow \infty} V((i+L P) h,(j+L Q) k)=W(i h-j h P / Q),
$$

exists, and

$$
0<W(i h-j h P / Q)<1, \forall i, \forall j \geq 0 .
$$


Proof. We have from (2.55)

$$
\begin{aligned}
& V((i+P) h,(j+Q) k)=\lim _{m \rightarrow \infty} v\left((i+P) h+\varphi^{m},(m+j+Q) k\right) \\
& \quad=\lim _{m \rightarrow \infty} v\left(i h+\left(P h+\varphi^{m}-\varphi^{m+Q}\right)+\varphi^{m+Q},(m+j+Q) k\right) \\
& \quad \leq \lim _{m \rightarrow \infty} v\left(i h+\varphi^{m+Q},(m+j+Q) k\right)=V(i h, j k) .
\end{aligned}
$$

Similarly, we prove that the sequence $(2.56)$ is decreasing. It then follows that the limit (2.57) exists, since the sequence is decreasing and bounded below by zero. Then from (2.57),

$$
\begin{aligned}
& W(i h-j h P / Q)=\lim _{L \rightarrow \infty} V((i+L P) h,(j+L Q) k) \\
& \quad=\lim _{L \rightarrow \infty}\left\{\lim _{m \rightarrow \infty} v\left((i+L P) h+\varphi^{m},(j+L Q+m) k\right)\right\} \\
& \quad=\lim _{L \rightarrow \infty}\left\{\lim _{m \rightarrow \infty} v\left(i h+\left(\alpha_{L}^{m}\right)+\varphi^{m+L Q},(j+L Q+m) k\right)\right\} .
\end{aligned}
$$

Since by Lemma 8 ,

$$
-2 h \leq \alpha_{L}^{-}
$$

it follows that there exists a subsequence $\left\{\alpha_{L}^{m(n)}\right\}, \forall n \geq 1$, such that

$$
\begin{gathered}
\alpha_{L}^{m(n)} \geq-3 h, \forall n \geq 1, \\
\alpha_{L}^{m(n)} \rightarrow \alpha_{L}^{-}, n \rightarrow \infty .
\end{gathered}
$$

Then, from (2.58), (2.59) and (2.60),

$$
\begin{aligned}
& W(i h-j h P / Q) \\
& \quad=\lim _{L \rightarrow \infty}\left\{\lim _{n \rightarrow \infty} v\left(i h+\left(\alpha_{L}^{m(n)}\right)+\varphi^{m(n)+L Q},(j+L Q+m(n)) k\right\}\right. \\
& \quad \geq \lim _{L \rightarrow \infty}\left\{\lim _{n \rightarrow \infty} v\left(i h-3 h+\varphi^{m(n)+L Q},(j+L Q+m(n)) k\right\}\right. \\
& \quad=\lim _{L \rightarrow \infty}\{V(i h-3 h, j k)\}=V(i h-3 h, j k)>0,
\end{aligned}
$$

by Lemma 5 . Similarly, we prove that

$$
W(i h-j h P / Q)<1 .
$$

Proof of Theorem 7 (cont). The case when

$$
\alpha_{1}^{-}=0 \leq \alpha_{1}^{+},
$$

is treated similarly. The difference equation (2.49) follows from (2.48) and (2.30). We finally prove that $W$ is unique. Let

$$
i_{1}-j_{1} P / Q=i_{2}-j_{2} P / Q
$$

Then

$$
\left(i_{1}-i_{2}\right) Q=\left(j_{1}-j_{2}\right) P .
$$

Since $(P, Q)=1$, we conclude that there exists a number $M$, such that

$$
\left(i_{1}-i_{2}\right)=M P \text { and }\left(j_{1}-j_{2}\right)=M Q .
$$


Then

$$
\begin{aligned}
& \lim _{L \rightarrow \infty} V\left(\left(i_{1}+L P\right) h,\left(j_{1}+L Q\right) k\right) \\
& \quad=\lim _{L \rightarrow \infty} V\left(\left(i_{2}+(L+M) P\right) h,\left(j_{2}+(L+M) Q\right) k\right) \\
& \quad=\lim _{L \rightarrow \infty} V\left(\left(i_{2}+L P\right) h,\left(j_{2}+L Q\right) k\right) .
\end{aligned}
$$

Theorem 8. Assume that $\gamma$, defined in Theorem 6 , is an irrational number. Then

$$
\sup _{h(i-\gamma j)<x} V(i h, j k)=W(x),-\infty<x<\infty,
$$

exists. $W$ is monotone and satisfies the difference equation

$W(x-\gamma h)=(1-2 \lambda) W(x)+\lambda W(x-h)+\lambda W(x+h)+k F(W(x)),-\infty<x<\infty$.

Proof. We first define $U$ from

$$
U(i h-j h \gamma)=V(i h, j k), \forall i, \forall j \geq 0 .
$$

This quantity is well defined, since if

$$
i_{1}-\gamma j_{1}=i_{2}-\gamma j_{2},
$$

then

$$
i_{1}-i_{2}=\gamma\left(j_{1}-j_{2}\right),
$$

and since $\gamma$ is irrational, it follows that

$$
i_{1}=i_{2} \text { and } j_{1}=j_{2} \text {. }
$$

Lemma 11. $U$ defined in (2.61) satisfies

$$
\begin{aligned}
& U(i h-\gamma j h-\gamma h)=(1-2 \lambda) U(i h-\gamma j h)+\lambda U(i h-h-\gamma j h) \\
& +\lambda U(i h+h-\gamma j h)+k F(U(i h-\gamma j h)), \forall i, \forall j \geq 0 .
\end{aligned}
$$

Proof.

$$
\begin{aligned}
& U(i h-\gamma j h-\gamma h)=U(i h-\gamma(j+1) h)=V(i h,(j+1) k) \\
& \quad=(1-2 \lambda) V(i h, j k)+\lambda V(i h-h, j k)+\lambda V(i h+h, j k)+k F(V(i h, j k)) \\
& \quad=(1-2 \lambda) U(i h-\gamma j h)+\lambda U(i h-h-\gamma j h)+\lambda U(i h+h-\gamma j h) \\
& \quad+k F(U(i h-\gamma j h) .
\end{aligned}
$$

Lemma 12. $U$ is monotone, i.e.,

$$
U\left(\left(i_{1}-\gamma j_{1}\right) h\right) \leq U\left(\left(i_{2}-\gamma j_{2}\right) h\right) \text { if } i_{1}-\gamma j_{1}<i_{2}-\gamma j_{2} .
$$

Proof. We consider first the case

$$
i_{2}=j_{2}=0 \text {. }
$$

It is then sufficient to prove that

$$
V(i h, j k) \leq V(0,0)=c \text { if } i<\gamma j, j \geq 0,
$$

or that

$$
\lim _{m \rightarrow \infty} v\left(i h+\varphi^{m},(m+j) k\right) \leq c=v\left(\varphi^{m+j},(m+j) k\right) \text { if } i<\gamma j, j \geq 0,
$$

(2.62) is satisfied if

$$
\liminf _{m \rightarrow \infty}\left(i h+\varphi^{m}-\varphi^{m+j}\right)<0, i<j \gamma, j \geq 0 .
$$


(2.63) is satisfied if

$$
\liminf _{m \rightarrow \infty}\left(\varphi^{m}-\varphi^{m+j}\right)<h\{1+[-j \gamma]\}, j \geq 0,
$$

where $[-j \gamma]$ denotes the integer part of $-j \gamma$, since

$$
i \leq-\{1+[-j \gamma]\} \text { if } i<j \gamma, j \geq 0,
$$

where $\gamma<0$. If for some $i$ and $j \geq 1$,

$$
\liminf _{m \rightarrow \infty}\left(\varphi^{m}-\varphi^{m+j}\right) \geq h\{1+[-j \gamma]\},
$$

then we derive from (2.45) and the definition of $\gamma$ that

$$
-j \gamma \geq 1+[-j \gamma], j \geq 1
$$

which is a contradiciton.

The proof is complete for $i_{2}=j_{2}=0$. The case $i_{1}=j_{1}=0$ is treated similarly. The general case is proved by reduction either to the case $i_{1}=j_{1}=0$ or to the case $i_{2}=j_{2}=0$.

Lemma 13. Let $\gamma$ be an irrational number. Then the set

$$
\{i-\gamma j ; \forall i, \forall j \geq 0\}
$$

is dense in the set of real numbers.

Proof. The result follows from fundamental properties of irrational numbers, see Hardy and Wright [3].

We then define

$$
W(x)=\sup _{h(i-\gamma j)<x} U((i-\gamma j) h)=\sup _{h(i-\gamma j)<x} V(i h, j k),
$$

which exist by Lemmas 12 and 13 .

Lemma 14. $W(x)$ defined in (2.64) satisfies the difference equation

$$
W(x-\gamma h)=(1-2 \lambda) W(x)+\lambda W(x-h)+\lambda W(x+h)+k F(W(x)) .
$$

Proof. Let $\left\{x_{n}\right\}, \forall n \geq 1$ be a sequence, such that

$$
x_{n}=h\left(i_{n}-\gamma j_{n}\right) ; x_{n}<x_{n+1}, \forall n \geq 1 \text { and } x_{n} \rightarrow x, n \rightarrow \infty .
$$

(2.65) then follows from Lemma 11 and the left continuity of $W$, due to Lemmas 12,13 and the definition (2.64).

Proof of Theorem 8 (cont.) The proof is completed by Lemmas 12 and 14 .

Theorem 9. Assume that the conditions of Theorem 6 are satisfied. Let

$$
\lim _{n \rightarrow \infty} \varphi^{n} / n=\gamma h
$$

Then

$$
\gamma=-\inf _{\alpha>0}\{\log [1-2 \lambda+k+\lambda(\exp (-\alpha h)+\exp (\alpha h))] /(\alpha h)\} .
$$

Proof. The result follows from Theorems 2, 6, 7, 8 and Lemma 15 below. 
Lemma 15. Let $\gamma$ be a real number. The difference equation

$$
Z(x-\gamma h)=(1-2 \lambda) Z(x)+\lambda Z(x-h)+\lambda Z(x+h)+k F(Z(x)),
$$

where

$$
\lim _{x \rightarrow-\infty} Z(x)=0 \text { and } \lim _{x \rightarrow \infty} Z(x)=1,
$$

has no measurable solution $Z(x)$, such that $0<Z(x)<1$, if

$$
0 \leq-\gamma<r
$$

where $r$ is defined by

$$
r=\inf _{\alpha>0}\{\log [1-2 \lambda+k+\lambda(\exp (-\alpha h)+\exp (\alpha h))] /(\alpha h)\} .
$$

Proof. Let $Z(x)$ be a measurable function, such that $0<Z(x)<1$, which satisfies (2.66) and (2.67). First, introduce a new variable $t$ defined by

$$
t=-x,
$$

and a new function $W$,

$$
W(t)=Z(-t)=Z(x)
$$

Then, from (2.66)

$$
\begin{aligned}
& W(t+\gamma h)=(1-2 \lambda) W(t)+\lambda W(t-h)+\lambda W(t+h) \\
& \quad+k F(W(t)),-\infty<t<\infty .
\end{aligned}
$$

Introduce the Laplace transform

$$
W_{L}(s)=\int_{0}^{+\infty} W(t) \exp (-s t) d t, s>s_{0},
$$

where $s$ is a real number. The definition of $W_{L}$ in (2.69) then implies that $W_{L}(s)$ is a decreasing and continuous function of $s$ for $s>s_{0}$, where

$$
s_{0}=\inf \{s ; W(s)<\infty\} .
$$

$s_{0}$ is either $-\infty$ or finite. Then, it follows that

$$
W_{L}(s) \rightarrow \infty, s_{0}<s \rightarrow s_{0},
$$

since $W_{L}(s)$ is continuous. We have

$$
W_{L}(s)<\infty \text { for } s>0,
$$

and we conclude that

$$
s_{0} \leq 0 \text {. }
$$

Transforming the difference equation (2.68) gives

$$
P(s) W_{L}(s)=Q(s)+R(s), s>s_{0},
$$


where

$$
\begin{aligned}
& P(s)=\exp (s \gamma h)-(1-2 \lambda)-k-\lambda(\exp (s h)+\exp (-s h)) \\
& Q(s)=-\exp (s \gamma h) \int_{\gamma h}^{0} W(t) \exp (-s t) d t \\
& +\lambda\left\{\exp (-s h) \int_{-h}^{0} W(t) \exp (-s t) d t-\exp (s h) \int_{0}^{h} W(t) \exp (-s t) d t\right\}, \\
& \text { (2.72) } R(s)=k \int_{0}^{\infty}\{F(W(t))-W(t)\} \exp (-s t) d t .
\end{aligned}
$$

Assume that

$$
0 \leq-\gamma<r
$$

Then, it follows that

$$
P(s) \neq 0, s<0,
$$

since if

$$
P\left(-s_{1}\right)=0, s_{1}>0
$$

then it follows from $(2.70)$ that

$$
\exp \left(-\gamma s_{1} h\right)=1-2 \lambda+k+\lambda\left(\exp \left(s_{1} h\right)+\exp \left(-s_{1} h\right)\right),
$$

which implies

$$
-\gamma=\log \left[1-2 \lambda+k+\lambda\left(\exp \left(-s_{1} h\right)+\exp \left(s_{1} h\right)\right)\right] /\left(s_{1} h\right), s_{1}>0
$$

which contradicts the assumption (2.73). We also have from (2.70) that

$$
P(0)=-k
$$

and

$$
\lim _{s \rightarrow-\infty} P(s)=-\infty
$$

since

$$
0 \leq-\gamma<r \leq 1
$$

Then, there exists a number $\delta>0$, such that

$$
\sup _{s \leq \delta} P(s)<0 .
$$

Division by $P(s)<0, s \leq \delta$, gives

$$
W_{L}(s)=Q(s) / P(s)+R(s) / P(s), s_{0}<s \leq \delta,
$$

or equivalently,

$$
\begin{aligned}
& \int_{0}^{\infty} \exp (-s t) W(t)\{1+(k / P(s))[1-F(W(t)) / W(t)]\} d t \\
& \quad=Q(s) / P(s), s_{0}<s \leq \delta,
\end{aligned}
$$

since $W(t)>0$ by assumption. There exists a constant $T>0$, such that

$$
\{1+(k / P(s))[1-F(W(t)) / W(t)]\}>0.5, T<t, s \leq \delta,
$$

since

$$
\lim _{t \rightarrow+\infty} F(W(t)) / W(t)=1
$$

and

$$
\sup _{s \leq \delta} P(s)<0 .
$$


Then for $s_{0}$ finite

$$
\begin{aligned}
& \int_{T}^{\infty} \exp (-s t) W(t)\{1+(k / P(s))[1-F(W(t)) / W(t)]\} d t \\
& \rightarrow \infty, s_{0}<s \rightarrow s_{0},
\end{aligned}
$$

Since $Q(s) / P(s)$ is bounded, we have found a contradiction. Similarly, we find a contradiction if $s_{0}=-\infty$, since

$$
\{1+(k / P(s))[1-F(W(t)) / W(t)]\}>0.5
$$

for $s$ sufficiently small, since

$$
P(s) \rightarrow-\infty, s \rightarrow-\infty .
$$

\section{Discussion AND CONClusions}

(1) The discrete analysis of the flame velocity, in particular Theorem 9, does not require a complete analysis of the existence of traveling wave solutions to the discrete equation. The reason is that a discrete version of Theorem 12 in Kolmogorov, Petrowsky and Piskunow [4, which implies strict mononicity for $V$, is replaced by a weaker condition (Lemma 6 ).

(2) The KPP-theory was proved for a simple model problem (Fisher's equation). In turbulent flame propagation we have models for the mean reaction rate, which satisfy the KPP-conditions (1.3)-(1.5); see Hakberg [2]. We may therefore expect that an extended KPP-theory is valid but the flame equations are too complex for a mathematical analysis. Validation of an extended KPP-theory is difficult for several reasons. First, we have errors due to finite values of $h$ and $k$. Second, numerical experiments suggest that $\left(\varphi^{n+1}-\varphi^{n}\right) / n k$ fluctuates and does not converge as $n$ tends to infinity. This paper suggests that $\lim _{n \rightarrow \infty} \varphi^{n} / n k$ is determined from numerical experiments and compared with a discrete KPP-theory as part of a validation procedure for the continuous equations.

\section{REFERENCES}

[1] Fisher, R. A. (1937), "The wave of advance of advantageous genes", Ann. Eugenics, 7 , 355369.

[2] Hakberg, B. (1997), "A critical study of the Bray-Moss-Libby model", Combustion Science and Technology, 125, 25-45.

[3] Hardy, G. H., Wright, E. M., An Introduction to the Theory of Numbers, Oxford, 1954. MR0067125(16:673c)

[4] Kolmogorov, A. N., Petrowsky, I. G., and Piskunow, N. S. (1937), "Study of the diffusion equation with growth of the quantity of matter and its application to a biology problem", Bull. Moskov. Gos. Univ. Math. Mekh., 1, no. 6, 1-25 (Russian); English translation in Dynamics of Curved Fronts, Pelcé, P. (editor), Academic Press, 1988, 105-130.

[5] Weinberger, H. F. (1982), "Long-time behaviour of a class of biological models", SIAM J. Math. Anal., 13, no. 3, 353-396. MR653463 (83f:35019)

[6] Zinner, B., Harris, G., and Hudson, W. (1993), "Travelling wave fronts for the discrete Fisher's equation", J. Differential Eqs., 105, 46-62. MR.1237977 (94k:39034)

Department of Mathematical Sciences, Chalmers and Gothenburg University, S-41296 Goteborg, Sweden

E-mail address: hakberg@chalmers.se 